\title{
Acute Megakaryocytic Leukemia with or Without Acquired Trisomy 21 in 15 Pediatric Patients
}

\author{
Wenzhi ZHANG \\ Huazhong University of Science and Technology \\ Hui LI \\ Huazhong University of Science and Technology \\ Jingzhen LIU \\ The Central Hospital of Enshi Tujia and Miao Autonomous Prefecture \\ Jiawei XU \\ Huazhong University of Science and Technology \\ Jinjin HAO \\ Huazhong University of Science and Technology \\ Hongbo CHEN \\ Huazhong University of Science and Technology \\ Xiaoyan WU ( $\nabla$ xwu@hust.edu.cn ) \\ Huazhong University of Science and Technology \\ Hui YU \\ Huazhong University of Science and Technology
}

\section{Research Article}

Keywords: Acute megakaryocytic leukemia, Acquired trisomy 21, Cytogenetics, Prognostic factors, Children

Posted Date: November 24th, 2020

DOI: https://doi.org/10.21203/rs.3.rs-107199/v1

License: (c) (i) This work is licensed under a Creative Commons Attribution 4.0 International License. Read Full License 


\section{Abstract}

The knowledge of clinical characteristics and prognosis of pediatric acute megakaryocytic leukemia (AMKL) with or without acquired +21 was limited. We reported 15 AMKL pediatric patients without Down Syndrome (four cases with acquired +21 and 11 cases without acquired +21 ) with the clinical manifestations, laboratory data, and prognosis. The clinical features and laboratory data between patients with acquired +21 and patients without acquired +21 are similar. As for prognosis, three of the 11 cases without acquired +21 obtained complete remission (CR) after 1 st induction. The median follow-up time of the 11 cases was 9 months. Among four cases with acquired +21 , one case gave up treatment during 1 st induction, one obtained $\mathrm{CR}$ after $1^{\text {st }}$ induction and was still alive after 49 months of follow-up. One case obtained CR after $2^{\text {nd }}$ induction and was still alive for 15 months of follow-up after bone marrow transplantation, the other patient was planning for allogeneic hematopoietic stem cell transplantation (HSCT) without CR. The median follow-up time of the four cases was 12 months. None relapsed in our study. In conclusion, acquired trisomy 21 may not be an indicator for poor prognosis. Cytogenetics analysis can help us for diagnosis stratification, prognostic judgment and individualized treatment of AMKL.

\section{Introduction}

AMKL is a rare subtype of pediatric acute myeloid leukemia (AML), characterized by abnormal megakaryoblasts that express platelet-specific surface glycoprotein. Patients with AMKL often presents with low blood cell count, especially thrombocytopenia. AMKL is biologically heterogeneous leukemia with special clinical and biological features, and it always carries a poor overall prognosis[1]. AMKL is extremely rare in adults, occurring in only $1 \%$ AML patients. In contrast, it comprises between $4 \%$ and $15 \% \mathrm{AML}$ patients in children[2]. The disease is generally divided into two major subgroups: AMKL in patients with Down syndrome (DS-AMKL) and without DS (non-DS-AMKL). Bone marrow biopsy of AMKL frequently demonstrates extensive myelofibrosis that often makes aspiration in these patients difficult. As a result of low incidence and difficult aspiration, AMKL is easy to be misdiagnosed or never diagnosed. AMKL is also characterized by various chromosomal abnormalities that are frequently associated with complex karyotypes and acquired clonal chromosome aberrations. Of which, the gain of chromosome 21 is one of the most commonly acquired trisomy in children with AMKL[3-6]. In addition, acquired trisomy 21 is a common chromosomal aberration in non-DSAMKL. There are limited knowledge and controversies about the clinical characteristics and prognosis of AMKL with acquired trisomy 21 [4, 7, 8], and here we report 11 cases of AMKL without acquired +21 and four cases of AMKL with acquired +21 to enhance the knowledge of this field.

\section{Results}

\section{Clinical characteristic}

From January 2013 to June 2020, 11 patients were diagnosed as AMKL without acquired trisomy 21, four cases were diagnosed as AMKL with acquired trisomy 21. None was diagnosed with central nervous system leukemia. Females were more than males, with a male to female ratio of 1:1.5. The median onset age of our patients was 22 months (range, 8-37months). All patients in our study presented thrombocytopenia initially, among which 6 cases had severe thrombocytopenia. 13 patients had elevated lactate dehydrogenase, suggesting a heavy tumor burden of AMKL patients. Most patients presented difficult bone marrow aspiration as a result of myelofibrosis. The clinical manifestations and laboratory data of patients are showed in Table 1.

\section{Morphology:}

The Bone marrow cell morphology of most patients with AMKL presented abnormal megakaryocyte proliferation, which is dominated by primitive megakaryocyte, and abnormal small megakaryocytes (Fig.1).

\section{Immunophenotyping]}

Bone marrow flow cytometry immunotyping was performed in ten of 15 patients. CD41 and CD42 were positive in $80 \%$ of patients. and CD61 was positive in $90 \%$ of patients. In addition, CD33 (90\%), CD34 (50\%), CD36(60\%)凶CD117 (60\%), CD13 (60\%), HLA-DR (30\%), CD3 (0\%), CD56 (30\%), and CD7 (30\%) were noticed. The immunotyping of four patients with +21 are listed in Table 2.

\section{Fusion gene:}

Fusion gene detection was carried out in all the 15 patients, among which two cases only carried EVI 1 gene, two cases carried EVI1 and WT1 gene, one case carried EVI1, WT1 and MPL gene, one case carried EVI1, WT1 gene, and GATA1 mutation『and one case carried EVI1 gene and MLL/AF9. (Table 3).

\section{Cytogenetics analysis:}

All the samples were subjected to karyotype analysis, and three of them failed. Among 12 evaluable patients, normal karyotype(n=3,25\%), complex karyotype $(n=9,75 \%)$. Among the nine patients with complex karyotype, four patients had acquired trisomy 21 abnormalities (Table 3 ). 
In the 4 cases of AMKL with acquired trisomy 21 , one case gave up treatment during $1^{\text {st }}$ induction. The other case obtained $\mathrm{CR}$ after $1^{\text {st }}$ induction and achieved sustained CR after the whole chemotherapy. This patient was followed up for a total of 49 months and was still alive. The $3^{\text {rd }}$ patient with acquired trisomy 21 obtained CR after $2^{\text {nd }}$ induction. Subsequently, the patient received HSCT, then followed up for 15 months after HSCT, and still alive in continuous remission. The $4^{\text {th }}$ patient, who didn't get CR after $2^{\text {nd }}$ induction, was planning for HSCT.

Among 11 cases without +21 , two cases gave up before treatment, the remaining nine cases received chemotherapy. One case died on the 5 th day of $1^{\text {st }}$ induction. Three cases $(27.3 \%)$ obtained CR after $1^{\text {st }}$ induction. Two cases obtained CR after $2^{\text {nd }}$ induction. The remaining three cases received HSCT after chemotherapy without CR. The median follow-up time for the 11 cases was 9 months (range, 1 51 months). None of the patients relapsed (Fig.2).

\section{Discussion}

AMKL is a subtype of AML with high heterogeneity. It is usually divided into DS-AMKL and non-DS-AMKL. Compared with DS-AMKL and other types of AML, non-DS-AMKL has a worse outcome. Regarding non-DS-AMKL, there are limited knowledge and controversies about the clinical characteristics and prognosis of AMKL with acquired trisomy 21[4, 7-12].

Acquired chromosome 21 has been often detected in AML, which strongly suggests that acquired chromosome 21 maybe an important factor involving the development of leukemia [13, 14]. AML patients with complex karyotypes have a poor prognosis[15]. Furthermore, AMKL has a high incidence of chromosomal abnormalities, most of which are accompanied by complex karyotypes. And chromosome 21 is one of the most frequently involved chromosomes aberrant.[3-6, 12, 16, 17]. Among the ten patients detected in our study, complex karyotype was found in seven patients, and three of which were associated with a gain of chromosome 21 , which was consistent with the literature. It was found that the overexpression of 21 chromosomes is a key role in the development of AMKL in many studies.[17-19].

In this study, the male to female ratio of 1:1.5 is different from many studies that mentioned males are more common in AMKL[9, 10]. The clinical features of the patients in our study were similar to the other research $[6,8,10,12]$. As for the prognosis, the two patients with acquired +21 were alive disease-free. Among which one patient was diagnosed as AMKL with GATA1 mutation and gain of chromosome 21, and allogeneic HSCT was performed on the patient after remission following $2^{\text {nd }}$ induction. The patient was followed up for 15 months after HSCT and was in an event-free survival state. Her chromosomes analysis before and after chemotherapy are shown in Fig.3. Previous studies have suggested that acquired chromosome 21 might be an indicator for poor prognosis $[4,20]$. Compared to those early studies, however, many works in recent years demonstrated that gained chromosome 21 was not supposed to be an independent risk factor. This is possibly related to administration of intensified chemotherapy $[8,11,21]$. In addition, in Jana Schweitzer's study, all the six patients with AMKL and GATA1 mutations were in continuous remission after treatment, indicating a similar biology and treatment response of AMKL with acquired or constitutional trisomy 21 in conjunction with GATA1s (relatively favorable prognosis) [11].

GATA1 is related to the amplification of chromosome 21 , even if it is found in non-DS-AMKL [7, 17, 22, 23].GATA1 encodes a zinc-finger transcription factor that regulates the normal development of the erythroid, megakaryocytic, and basophilic/mast cell lineages, and is associated with transient myelopoiesis and DS-AMKL[24-26]. A recent study showed that GATA1 mutations can be detected in 11\% of non-DS-AMKL patients and that lowintensity chemotherapy may also be effective in these patients[27, 28]. Similarly, the ERG has recently been shown to play a role in hematopoietic stem cells as well as the development of the megakaryocytic lineage[2]. The ERG gene encodes a potent proto-oncogene and is expressed in hematopoietic stem cells as well as endothelial cells. Furthermore, many studies have demonstrated that ERG is involved in many chromosome translocations. It was assumed that the acquired gain of chromosome 21 or amplification of chromosome arm $21 \mathrm{q}$ is one mechanism contributing to increased ERG expression in AML[5].

In summary, non-DS-AMKL is a disease with a poor prognosis. With the improvement of diagnosis and intense chemotherapy, the survival of AMKL has been greatly improved. Chromosome 21 plays an important role in the occurrence and development of tumors, especially AMKL. In conclusion, acquired chromosome 21 may not be an indicator for poor prognosis. Therefore, cytogenetics analysis is helpful for AMKL risk stratification, prognostic judgment, and individualized treatment.

This study has some limitations. Although our longest follow-up time was 51 months, the time for observation in our study is still not long enough. It was found that half of patients live in disease-free without HSCT in our study, which is different from most studies. Additionally, the incidence of AMKL is very low and the number of patients is relatively limit in our work. Therefore, a longer follow-up period and larger sample sizes are required in the near future to overcome the limitations of this study, to get clearer idea of the patients' prognosis, and to verify the function of 21 chromosomes in AMKL and improve clinicians' understanding of AMKL.

\section{Methods}


The study was conducted in accordance with the principles of the Declaration of Helsinki, and the study protocol was approved by the ethics committee of Union Hospital Affiliated with Tongji Medical College of Huazhong University of Science and Technology. Because of the retrospective nature of the study, patient consent for inclusion was waived.

In total, we analyzed 15 AMKL pediatric patients without Down Syndrome (four cases with acquired +21 and 11 cases without acquired +21 ) between January 2013 and June 2020, including six males and nine females. All the patients were diagnosed with non-DS-AMKL referring to the 2008 revision of World Health Organization classification. Karyotype analysis was performed in 12 of the cases. Four patients were associated with acquired trisomy 21 aberration, five with complex karyotype without acquired trisomy 21 aberration, and three with normal karyotype.

\section{Immunophenotyping}

Eight-color fluorescent direct immunoassay was used to detect the expression of surface antigen. $>20 \%$ of antigen-expressing cells in immature myeloid cells were considered as positive.

\section{Fusion gene}

Fusion gene test on the $3 \mathrm{ml}$ bone marrow specimens with EDTA were performed by real-time polymerase chain reaction.

\section{Cytogenetics analysis}

The karyotypes were analyzed by G banding technique and described according to ISCN 2009 standard.

\section{Diagnostic criteria}

The diagnostic confirmation of AMKL was determined by morphology, immunophenotyping, and cytogenetics. The morphologic diagnosis was defined by the presence of $\geq 20 \%$ blasts in bone marrow, among which $50 \%$ are megakaryoblasts. The blasts usually show granular structure staining for PAS and negative staining for MPO. Besides, CD41 staining of bone marrow and peripheral blood may help with diagnosis. As a result of myelofibrosis, dry tap aspiration or dilution of bone marrow are common in AMKL. Hence, flow immunotyping often fails to work. The blasts of non-DS-AMKL usually express markers CD13, CD33, CD61 without CD34, while the blasts of DS-AMKL express markers CD7, CD11b, CD36. The aberrations of gain of chromosome 8 , gain of chromosome 21 , monosomy 7 , and $t(1 ; 22), t(11 ; 12)$ are common in AMKL.

\section{Treatment Protocol}

Patients received routine chemotherapy for AML. $1^{\text {st }}$ induction phase contains DAE (daunorubicin, cytarabine (Ara-C), and etoposide) or DAH (daunorubicin, Ara-C, Homoharringtonine) randomly. $2^{\text {nd }}$ induction phase contains IAE (idarubicin, Ara-C, and etoposide) or IAH (idarubicin, Ara-C, Homoharringtonine). Consolidation phase includes MA (Mitoxantrone, high-dose Ara-C)-HA (Homoharringtonine, Ara-C)-CLASP (Ara-C, Lasparaginase), and the chemotherapy dose was adjusted according to the risk level. At maintenance phase, patients were randomized to different treatment groups of Ara-C with 6 mercaptopurine (6-MP) or all-trans retinoic acid (ATRA) with 6-MP. Some patients with or without CR were offered HSCT.

\section{Response evaluation}

Evaluations of bone marrow aspirates were performed after $1^{\text {st }}$ and $2^{\text {nd }}$ inductions. The evaluation was conducted after each course of consolidation. During maintenance treatment, the evaluation was conducted every six months to the end of the chemotherapy. CR was defined as bone marrow with $<5 \%$ blasts and evidence of regeneration of normal hematopoietic cells, and the minimal resident disease (MRD) $\leq 10^{-4}$. Relapse was defined as the presence of $\geq 5 \%$ blasts in the bone marrow or extramedullary relapse. The last follow-up date was September 30,2020 .

\section{Abbreviations}

AMKL: acute megakaryocytic leukemia

CR: complete remission

HSCT: allogeneic hematopoietic stem cell transplantation

AML: acute myeloid leukemia

DS-AMKL: AMKL with Down syndrome

Non-DS-AMKL: AMKL without Down syndrome 
Ara-C: cytarabine

6-MP: 6 mercaptopurine

ATRA: all-trans retinoic acid

MRD: minimal resident disease

\section{Declarations}

\section{Data availability statement}

All data generated or analysed during this study are included in this published article.

\section{Acknowledgements}

This work was supported by Innovative Research Grant from Wuhan Science and Technology Department (No. 2019020701011503)

\section{Author contributions}

The study was originally and conceptually developed and supervised until its completion by Xiaoyan WU; Wenzhi ZHANG performed the data collection, data analyses and wrote the manuscript; Xiaoyan WU, Hongbo CHEN helped perform the analysis with constructive discussions. Hui LI, Jingzhen LIU, Hui YU, Jiawei XU, Jinjin HAO provided data of patients.

\section{Competing interests}

The authors declare no competing interests.

\section{Compliance with ethical standards}

The study was conducted in accordance with the principles of the Declaration of Helsinki, and the study protocol was approved by the ethics committee of Union Hospital Affiliated with Tongji Medical College of Huazhong University of Science and Technology. Because of the retrospective nature of the study, patient consent for inclusion was waived.

\section{References}

1. Vardiman, J.W., J. Thiele, D.A. Arber, R.D. Brunning, M.J. Borowitz, A. Porwit, et al., The 2008 revision of the World Health Organization (WHO) classification of myeloid neoplasms and acute leukemia: rationale and important changes.Blood. 114, 937-951(2009).

2. Gruber, T.A. and J.R. Downing, The biology of pediatric acute megakaryoblastic leukemia.Blood. 126, 943-9(2015).

3. Shu-Xu, D., Z. Shi-Xuan, W. Ying, C. Wen, L. Cheng-Wen, C. Yu-Mei, et al., Analysis on the laboratory examination characteristics in 22 patients with acute megakaryoblastic leukemia.Chin J Hematol. 037, 297-301(2016).

4. Yong-Ming, X., C. Zhi-Mei, W. Huan-Ping, L. Ji-Yu, X. Huan, Y. Yun-Biao, et al., 9 cases of acute leukemia with simple trisomy 21.Chin J Hematol. 31, 347-348(2010).

5. Simone, Weber, Claudia, Haferlach, Sabine, Jeromin, et al., Gain of chromosome 21 or amplification of chromosome arm 21q is one mechanism for increased ERG expression in acute myeloid leukemia.GENES, CHROMOSOMES \& CANCER,(2016).

6. Wen-Yu, Y., C. Xiao-Juan, L. Mei, W. Shu-Chun, L. Tian-Feng, and Z. Xiao-Fan, Analysis of clinical and biologic characteristics in pediatric acute megakaryoblastic leukemia.China Pediatr Blood Cance,(2008).

7. Ono, R., D. Hasegawa, S. Hirabayashi, T. Kamiya, K. Yoshida, S. Yonekawa, et al., Acute megakaryoblastic leukemia with acquired trisomy 21 and GATA1 mutations in phenotypically normal children.Eur J Pediatr. 174, 525-31(2015).

8. Yu-Juan, X., Y. Shui, L. Ai-Dong, W. Jun, Z. Ying-Xi, J. Yue-Ping, et al., Analysis of clinical features in 22 patients with pediatric acute megakaryoblastic leukemia.Journal of Clinical Pediatrics. 036, 834-839(2018).

9. Chao-Rong, W., W. Ya-Feng, G. Yu-Jie, Z. Jian-Wen, Z. Ying-Ying, S. Li-Li, et al., Clinical features and prognosis of acute megakaryocytic leukemia in children.Mod Med J China, 5-9(2019).

10. Marques-Piubelli, M.L., Acute megakaryoblastic leukemia with t(1;22)(p13.3;q13.1); RBM15-MKL1 mimicking hepatoblastoma in an infant: The role of karyotype in differential diagnosis.Pediatr Blood Cancer,(2019).

11. Schweitzer, J., M. Zimmermann, M. Rasche, C. von Neuhoff, U. Creutzig, M. Dworzak, et al., Improved outcome of pediatric patients with acute megakaryoblastic leukemia in the AML-BFM 04 trial.Annals of Hematology. 94, 1327-1336(2015).

12. Maarouf, N., S. Mahmoud, R. Khedr, L. Lehmann, K. Shaaban, S. Ibrahim, et al., Outcome of Childhood Acute Megakaryoblastic Leukemia: Children's Cancer Hospital Egypt 57357 Experience.Clin Lymphoma Myeloma Leuk. 19, e142-e152(2019). 
13. Izraeli, S., L. Rainis, L. Hertzberg, G. Smooha, and Y. Birger, Trisomy of chromosome 21 in leukemogenesis.Blood Cells, Molecules, and Diseases. 39, 156-159(2007).

14. Hai-Zhi, Y., C. Zhao, X. Si-Si, Y. Yi-Fang, and P. Hong-Ling, Analysis of Clinical Characteristics in 31 Acute Myeloid Leukemia Patients with Chromosome 21 Aberrations. Journal of Experimental Hematology,(2017).

15. CD., B., S. Liyanarachchi, M. K., H. Auer, T. SM., G.M.R. AS., et al., Acute myeloid leukemia with complex karyotypes and abnormal chromosome 21: Amplification discloses overexpression of APP, ETS2, and ERG genes.Proc Natl Acad Sci U S A. 101, p. 3915-3920(2004).

16. Masetti, R., V. Guidi, L. Ronchini, N.S. Bertuccio, F. Locatelli, and A. Pession, The changing scenario of non-Down syndrome acute megakaryoblastic leukemia in children. Crit Rev Oncol Hematol. 138, 132-138(2019).

17. Lopez, C.K., S. Malinge, M. Gaudry, O.A. Bernard, and T. Mercher, Pediatric Acute Megakaryoblastic Leukemia: Multitasking Fusion Proteins and Oncogenic Cooperations. Trends Cancer. 3, 631-642(2017).

18. Pelleri, M.C., A. Piovesan, M. Caracausi, A.C. Berardi, L. Vitale, and P. Strippoli, Integrated differential transcriptome maps of Acute Megakaryoblastic Leukemia (AMKL) in children with or without Down Syndrome (DS).BMC Med Genomics. 7, 63(2014).

19. De Marchi, F., M. Araki, and N. Komatsu, Molecular features, prognosis, and novel treatment options for pediatric acute megakaryoblastic leukemia.Expert Rev Hematol. 12, 285-293(2019).

20. De-Hong, N. and W. Da-Ben, Acute erythrocytic leukemia with simple trisomy 21: a case report and literature.Shangdong Medical Journal, 9798(2015).

21. Inaba, H., Y. Zhou, O. Abla, S. Adachi, A. Auvrignon, H.B. Beverloo, et al., Heterogeneous cytogenetic subgroups and outcomes in childhood acute megakaryoblastic leukemia: a retrospective international study.Blood. 126, 1575-1584(2015).

22. Wang, S.A. and R.P. Hasserjian, Acute Erythroleukemias, Acute Megakaryoblastic Leukemias, and Reactive Mimics: A Guide to a Number of Perplexing Entities.Am J Clin Pathol. 144, 44-60(2015).

23. Shin, M.-G., H.-W. Choi, H.-R. Kim, M.-J. Kim, H.-J. Baek, D.-K. Han, et al., Tetrasomy 21 as a sole acquired abnormality without GATA1 gene mutation in pediatric acute megakaryoblastic leukemia: A case report and review of the literature.Leukemia Research. 32, 1615-1619(2008).

24. Lee, W.Y., O.K. Weinberg, and G.S. Pinkus, GATA1 Is a Sensitive and Specific Nuclear Marker for Erythroid and Megakaryocytic Lineages.Am J Clin Pathol. 147, 420-426(2017).

25. Crispino, J.D. and M.S. Horwitz, GATA factor mutations in hematologic disease.Blood. 129, 2103-2110(2017).

26. Bourquin, J.P., A. Subramanian, C. Langebrake, D. Reinhardt, O. Bernard, P. Ballerini, et al., Identification of distinct molecular phenotypes in acute megakaryoblastic leukemia by gene expression profiling. Proc Natl Acad Sci U S A. 103, 3339-3344(2005).

27. McNulty, M. and J.D. Crispino, Acute Megakaryocytic Leukemia.Cold Spring Harb Perspect Med. 10(2020).

28. Terui, K., T. Toki, T. Taga, S. Iwamoto, T. Miyamura, D. Hasegawa, et al., Highly sensitive detection of GATA1 mutations in patients with myeloid leukemia associated with Down syndrome by combining Sanger and targeted next generation sequencing.Genes Chromosomes Cancer. 59, 160-167(2020).

\section{Tables}

Table 1. The clinical manifestations and laboratory data of patients at diagnosis

\begin{tabular}{|c|c|c|c|c|c|c|c|c|c|}
\hline \multirow{3}{*}{ Patients } & \multicolumn{6}{|c|}{ Clinical manifestations } & \multicolumn{3}{|c|}{ Laboratory data } \\
\hline & Anemia & Fever & Hemorrhage & Hepatomegaly & Splenomegaly & Lymphadenectasis & WBC & $\mathrm{HB}$ & PLT \\
\hline & & & & & & & $\mathrm{G} / \mathrm{L}$ & $g / L$ & $\mathrm{G} / \mathrm{L}$ \\
\hline $\begin{array}{l}\text { AMKL } \\
\text { without } \\
+21 \\
\text { n(\%) }\end{array}$ & 10(91) & $8(73)$ & $8(73)$ & $5(45)$ & $4(36)$ & $3(27)$ & $13.5 \pm 7.8$ & $76.1 \pm 23.3$ & $27.6 \pm 17.4$ \\
\hline $\begin{array}{l}\text { AMKL } \\
\text { with } \\
+21, \\
n(\%)\end{array}$ & $4(100)$ & $4(100)$ & $3(75)$ & $3(75)$ & $3(75)$ & $0(0)$ & $16.7 \pm 11.8$ & $86.3 \pm 4.1$ & $42.5 \pm 14.1$ \\
\hline
\end{tabular}

Abbreviations: AMKL, acute megakaryocytic leukemia. Data presented as mean \pm standard deviation

Table 2. Immunotyping results of four cases with acquired trisomy 21 


\begin{tabular}{|llllllllllllll|}
\hline patient & HLA-DR & CD3 & CD7 & CD13 & CD33 & CD34 & CD36 & CD41a & CD42b & CD56 & CD61 & CD117 \\
1 & - & - & - & + & + & + & - & - & $/$ & + & + & + \\
2 & - & - & + & + & + & - & + & + & + & - & + & + \\
3 & + & - & + & + & + & + & + & - & + & + & + & + \\
4 & + & - & + & + & + & + & - & + & + & - & + & + \\
\hline
\end{tabular}

"+": positive; "-": negative; "/": not complete the test.

Table 3. Cytogenetic analysis $\square$ fusion gene results and survival time of 15 cases

\begin{tabular}{|c|c|c|c|}
\hline Patient & Fusion gene & Karyotype & $\begin{array}{l}\text { Survival } \\
\text { time(months) }\end{array}$ \\
\hline 1 & _a & $53, X Y,+X,+Y,+4,+6,+19,+20,+21[18] / 46, X Y[2]$ & $49+$ \\
\hline 2 & $\begin{array}{l}\text { WT1LGATA10 } \\
\text { EVI1 }\end{array}$ & $47 \sim 49, \mathrm{XX},+21,+$ marl,+ mar2[cp20] & $20+$ \\
\hline 3 & - & $51, X Y,+6,+8,+14,+19,+21[20]$ & 2 \\
\hline 4 & MLL/AF9aEVI1 & $50, X Y,+6,+19,+21,+22[3] / 46, X Y,[17]$ & $4+$ \\
\hline 5 & - & $56-57, X X,+1,+6,+7+10,+19,+22$, inc $[\mathrm{cp} 2] / 46, X X[7]$ & $35+$ \\
\hline 6 & EVI1 & $60, X Y,+Y,+1,+2,+4,+5,+6,+7,+7,+8,+10,+14, \operatorname{add}(16)(p 13),+19,+19,+22[20]$ & $5+* c$ \\
\hline 7 & EVI11WT1 & $46, X X[4]$ & $14+$ \\
\hline 8 & EVI10WT10MPL & $46, X Y$ & $13+$ \\
\hline 9 & WT10EVI1 & 67-73,XX,inc[cp4]/46,XX[16] & $51+$ \\
\hline 10 & - & $\operatorname{Add}(X q), \operatorname{der}(4), \operatorname{del}(5 p), \operatorname{der}(7), \operatorname{der}(9), \operatorname{der}(11), \operatorname{add}(12 p),-13,-17, \operatorname{add}(17 q)$ & $12+$ \\
\hline 11 & - & $46, X Y[20]$ & 1 \\
\hline 12 & - & $j^{b}$ & $12+$ \\
\hline 13 & - & / & 4 \\
\hline 14 & EVI1 & / & 5 \\
\hline 15 & - & $\begin{array}{l}\text { 46,XX,der(3)?t(3;6)(p11;q11), del(6), der(7)t(3;7)(p14;q36), add(15)(p11), add(19)(p13) } \\
{[10 \rrbracket}\end{array}$ & $7+$ \\
\hline
\end{tabular}

a: "-": negative; b: "/": not complete the test; c: “*”: The case was lost to follow up.

\section{Figures}




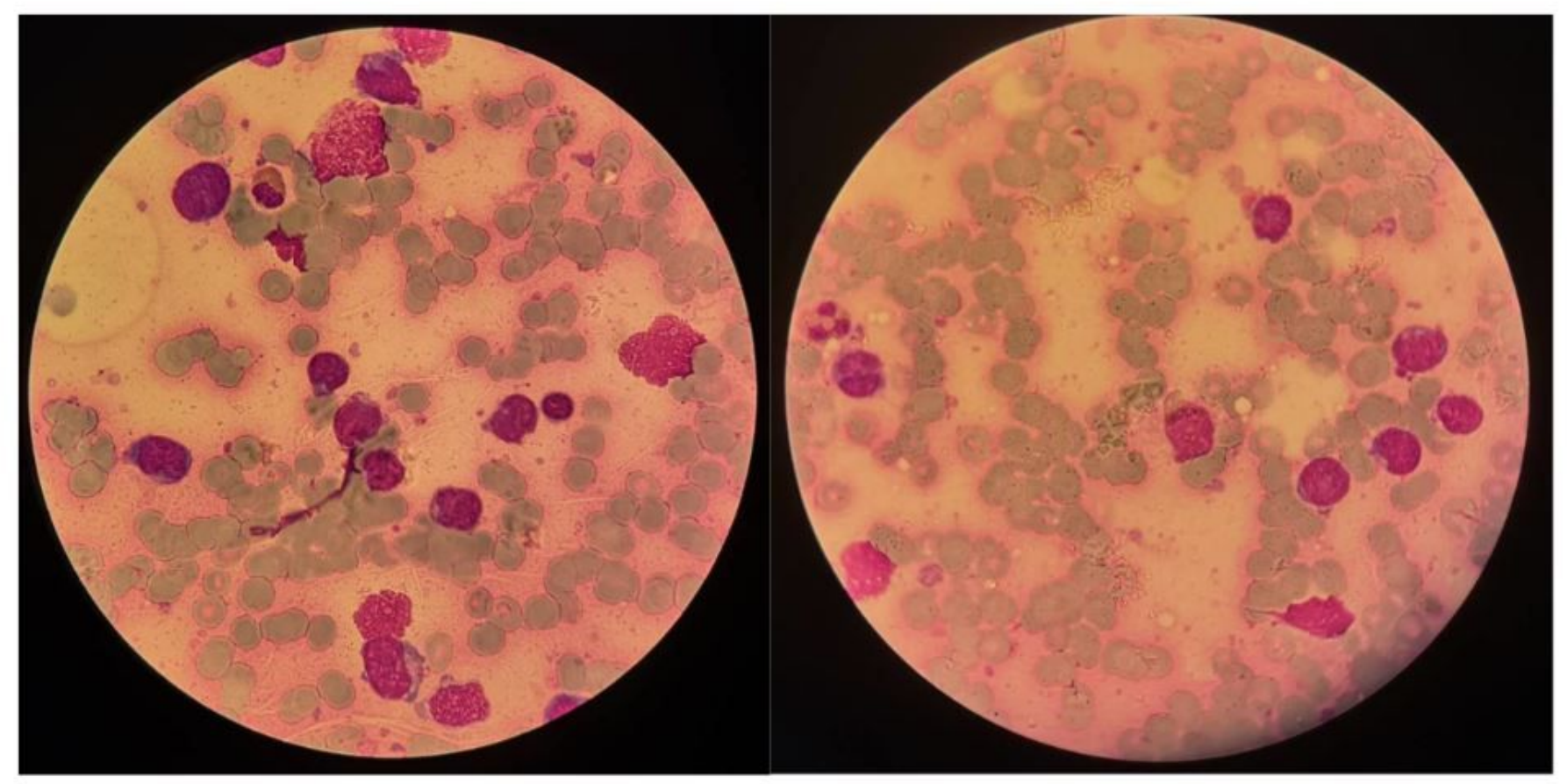

\section{Figure 1}

Bone marrow cell morphology of a patient diagnosed as AMKL without +21

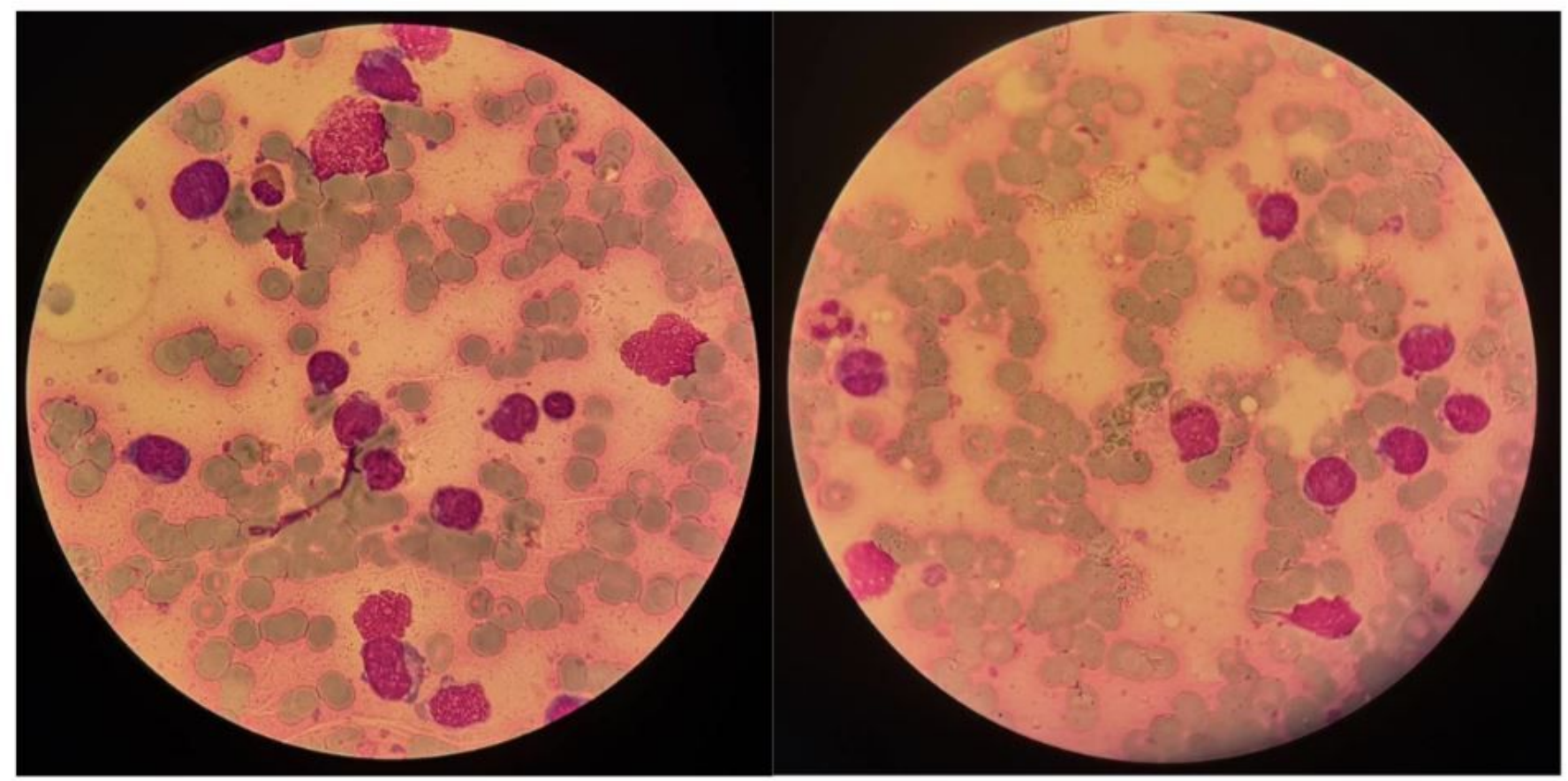

Figure 1

Bone marrow cell morphology of a patient diagnosed as AMKL without +21 


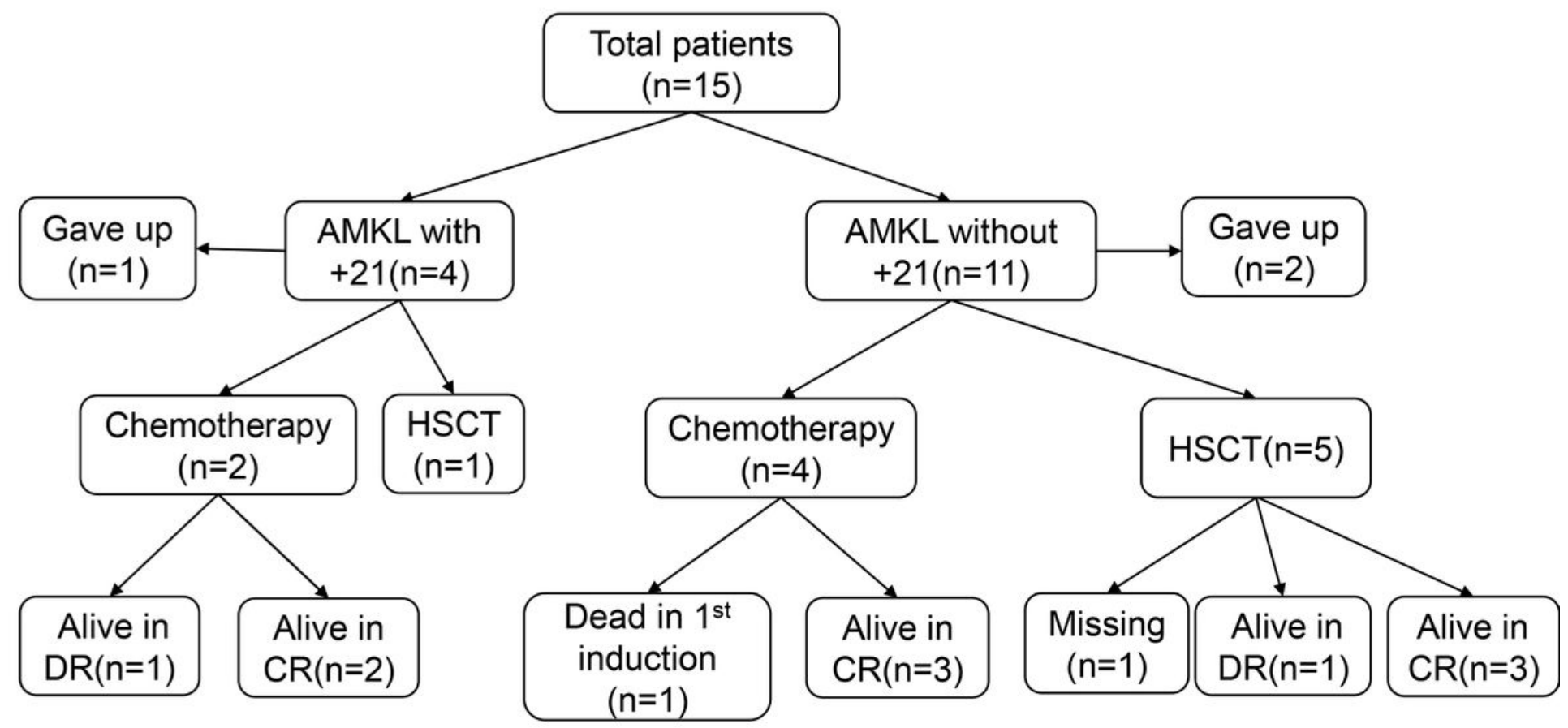

Figure 2

Outcomes of 15 patients Abbreviations: AMKL, acute megakaryocytic leukemia; HSCT, hematopoietic stem cell transplantation; CR, complete remission. 


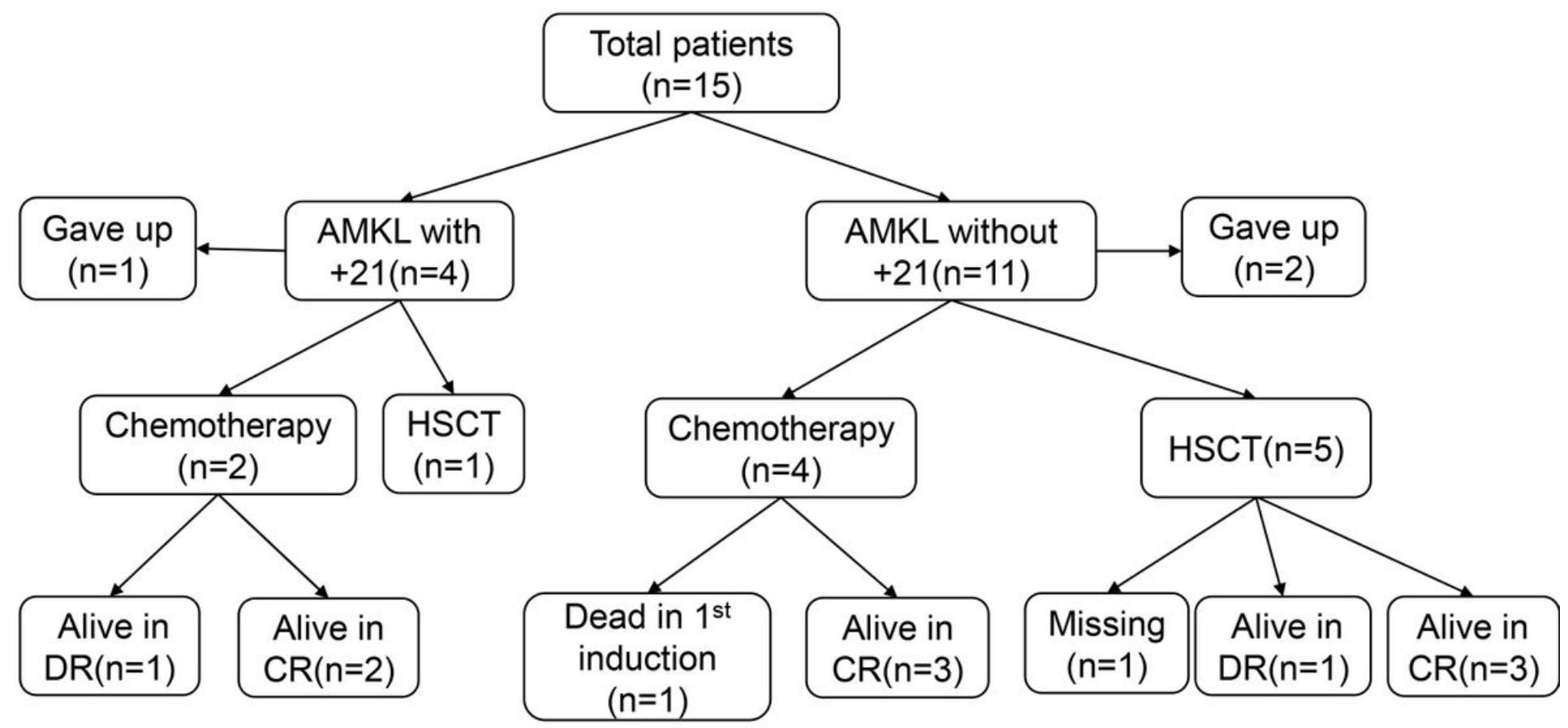

Figure 2

Outcomes of 15 patients Abbreviations: AMKL, acute megakaryocytic leukemia; HSCT, hematopoietic stem cell transplantation; CR, complete remission. 
a

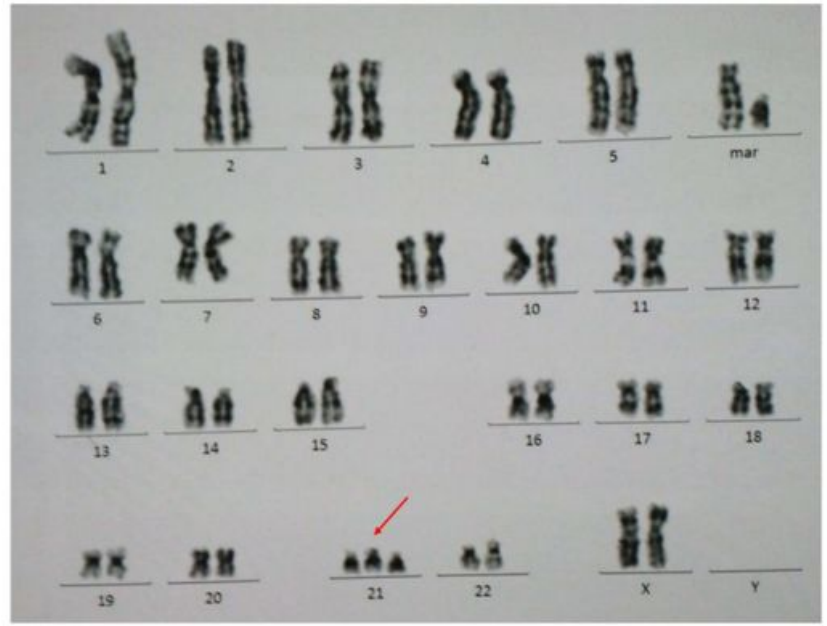

b

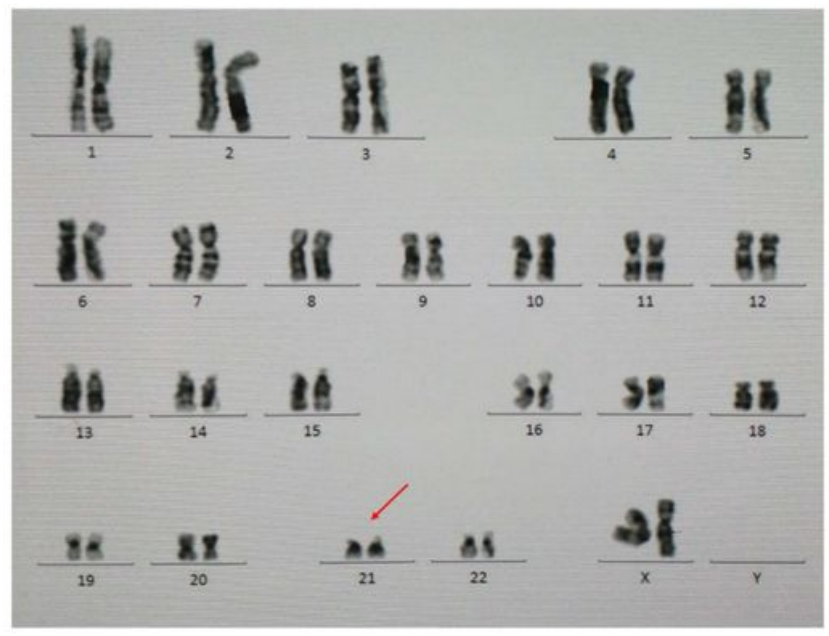

Figure 3

Karyotype of patient 2. a. At initial stage, chromosome analysis showed 47 49,XX,+21,+marl,+mar2[cp20]. b. At remission stage, chromosome analysis showed $46, \mathrm{XX}$ 
a

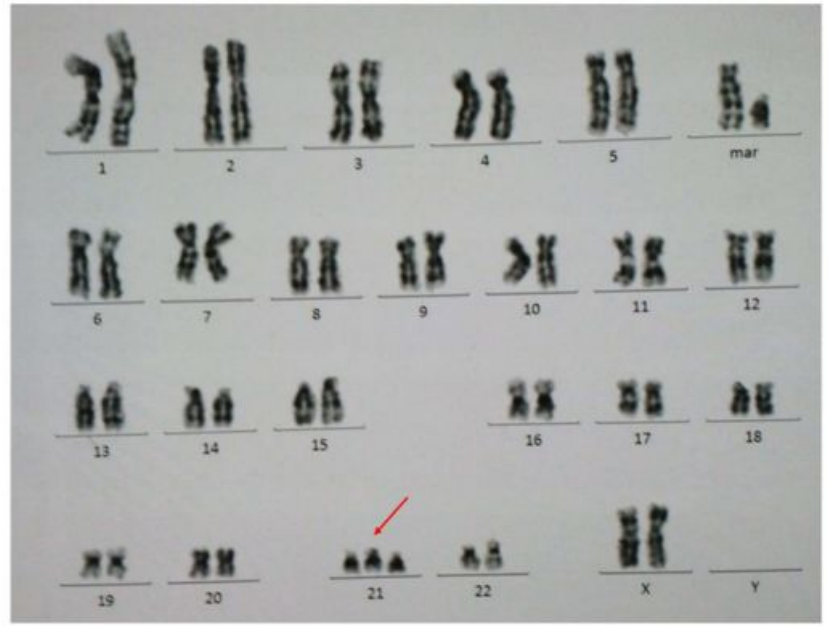

b

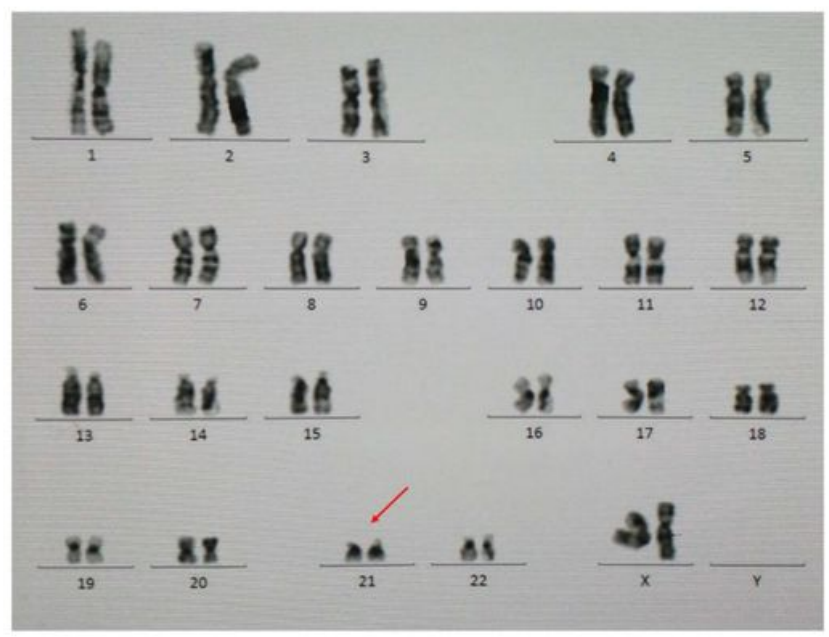

Figure 3

Karyotype of patient 2. a. At initial stage, chromosome analysis showed 47 49,XX,+21,+marl,+mar2[cp20]. b. At remission stage, chromosome analysis showed $46, \mathrm{XX}$ 